Sharif University of Technology
Scientia Iranica
SCIENTIA
IRANICA
Transtions E: Industrial Engineering
www.scientiairanica.com

\title{
Cost-effectiveness analysis of the mobile phone manufacturing sector by using a novel fuzzy multi-objective programming model
}

\author{
T.S. Su ${ }^{\mathrm{a}}$, C.C. Wu ${ }^{\mathrm{b}, *}$ and C.H. $\mathrm{Lin}^{\mathrm{a}}$ \\ a. Department of Industrial Management, National Pingtung University of Science and Technology, 1 Hsueh-Fu Rd., Nei Pu Hsiang, \\ Pingtung, 912, Taiwan. \\ b. Department of Information Management, I-SHOU University, No.1, Sec. 1, Syuecheng Rd., Dashu District, Kaohsiung 84001, \\ Taiwan.
}

Received 3 May 2014; received in revised form 24 March 2015; accepted 16 June 2015

\author{
KEYWORDS \\ Mobile phone; \\ Fuzzy multi-objective \\ programming; \\ S-curve membership \\ function; \\ Cost effectiveness; \\ Production planning; \\ Manufacturer/supplier.
}

\begin{abstract}
This work presents a novel Fuzzy Multi-Objective Programming (FMOP) model with a modified S-curve membership function capable of solving integrated multicomponent, multi-supplier, and multi-time-period production planning problems by using fuzzy objectives for the mobile phone manufacturing sector. The proposed model attempts to minimize total manufacturing, total inventory holding, and total penalty costs, simultaneously, in relation to manufacturer/supplier capacity and warehouse space. Additionally, the proposed model provides a systematic means of facilitating the fuzzy decision-making process and enabling decision makers to interactively adjust the search direction during the solution procedure in order to obtain the preferred satisfactory solution of a Decision Maker (DM). Moreover, adequacy of the proposed model is demonstrated based on an implementation design involving several scenarios of manufacturing production system for mobile phones. Analytical results provide a valuable reference for decision managers attempting to more thoroughly understand the systematic analysis and potential of cost-effective production planning.

(C) 2016 Sharif University of Technology. All rights reserved.
\end{abstract}

\section{Introduction}

Widely viewed as extremely competitive, the mobile phone market is characterized by its short life cycle, high utility, specialization, quick response, and low manufacturing costs [1]. To reduce production cycle time in order to achieve low-cost manufacturing, enterprises have changed production modes by adopting the homemaking mode for core technologies, while incorpo-

*. Corresponding author. Tel.: +8867 6577711 \#6580; Fax: +886 76578491

E-mail addresses: tyson@mail.npust.edu.tw (T.S.Su); chinchunw@isu.edu.tw (C.C.Wu);

charlielin0601@gmail.com (C.H.Lin) rating outsourcing modes for non-core technologies [24]. Developing cost-effective manufacturing outsourcing systems requires that mobile phones manufacturing enterprises maintain beneficial to make-or-buy ratios, focus solely on core operations, and maximize flexibility by focusing manufacturing on core operations. For instance, with mobile phones, core technologies (e.g., design and chip production) are homemade, while nonecore technologies (e.g., assembly and test operations) are outsourced. Decisions involving time and quantity for homemaking and outsourcing profoundly impact inventory holding and penalty costs. For instance, although inventory holding costs increase if the production time is too short, the occurrence opportunity of the penalty cost may be reduced. Conversely, although 
inventory holding costs may decrease if the production time is too long, the occurrence opportunity of the penalty cost might increase. Therefore, when subject to trade-off, the decision of production time and quantity is a complex problem involving a production plan on how to determine the most appropriate production time and quantity for mobile phones without incurring too many inventory holding and penalty costs.

Production planning decisions and input data or parameters (e.g., production costs) involve many uncertainties that must be resolved when evaluating the production costs in mobile phone manufacturing [5]. Additionally, related parameters (e.g., forecasting demand, supplier capacity, warehouse space, and objective function) are often imprecise owing to incomplete information. Production managers must consider multiple objectives, simultaneously, such as minimizing the total manufacturing cost, minimizing the inventory holding cost, and minimizing the penalty cost. However, most related research focuses on a single objective $[1,6]$. These factors produce an objective function with fuzziness. For instance, the objective function of the total annual manufactured costs may be $\$ 7$ million (Taiwan dollar), annual holding costs may be $\$ 0.3$ million (Taiwan dollar), and annual penalty costs may be $\$ 0.1$ million (Taiwan dollar). This imprecision warrants the need for a set of fuzzy multi-objective models to produce a set of compromising solutions. Peidro and Vasant [7] compared various membership functions, including linear, piecewise linear, exponential, and hyperbolic membership functions. In particular, S-curve membership function is sufficiently flexible to describe the vagueness in fuzzy parameters for production planning problems [7-10]. Therefore, the proposed Fuzzy Multi-Objective Programming (FMOP) model uses the fuzzy theory developed by the modified S-curve membership function [11]. This model can help Decision Makers (DMs) to systematically analyze the cost-effectiveness of production-planning schemes for mobile phones. This work focuses on the following objectives:

1. Analyzing production-planning decisions for mobile phone manufacturing under a fuzzy environment;

2. Devising an FMOP model that incorporates production, inventory holding, and penalty costs;

3. Developing a cost-effective model for evaluating the production planning of mobile phone manufacturing;

4. Illustrating the development process and solution through the FMOP model on an actual case of mobile phone manufacturing in Taiwan.

The rest of this paper is organized as follows: Section 2 reviews the pertinent literature. Then, Section 3 formulates the fuzzy multi-objective mobile phones production-planning decisions model. Next, Section 4 evaluates feasibility of the proposed FMOP model in a case study of a mobile phone manufacturing firm. Conclusions are finally drawn in Section 5 along with recommendations for future research.

\section{Literature review}

The global market demand for mobile phones has rapidly expanded in recent years. Market demand can be categorized as high-price mobile phones with a high profit or low-price mobile phones with a large amount of demand volume. To satisfy the large demand of a highly selected customer, mobile phone manufacturing has shifted towards higher quality, shorter delivery times, and lower product costs [5]. However, as technologies and components are increasingly standardized and modularized, new mobile phone manufacturers have begun outsourcing core technologies and product modules [12]. To shorten the delivery cycle and reduce production costs, manufacturers normally adopt homemaking and R\&D for core technologies and core production while outsourcing non-core technologies for vendors to create [13,14]. For instance, Nokia mobile phones define its core competence as developing, designing, and manufacturing mobile phones [15]. Nokia and Ericsson have adopted this pattern for outsourcing their production to a joint producer in order to optimally exploit economies of scale [16].

Owing to intensive price competition, the production network of the mobile phone supply chain is characterized by increasing outsourcing, vertical reintegration by contract manufacturers, and production shifting to countries with low labor costs. For instance, Nokia, Motorola, and Ericsson are the largest outsourcers in the mobile phone industry with $15 \%$ $66 \%$ of their production outsourced $[17,18]$. Olhager et al. [19] introduced a mobile phone supply chain, including raw material producers, material fabricators, factories, distributors, retail shops, and consumers. Mobile phones consist of many components, including chips, Printed Circuit Boards (PCBs), power amplifiers, memories, panels, optical parts, electronic parts, cells, as well as keypads and cases. Many manufacturers supply the components. For instance, Sanyo supplies batteries, Exel provides logistical services, Ibiden provides Printed Wiring Boards (PWBs), Verso supplies accessories, Elcoteq provides PWBs and engines, and Foxconn provides low end phones [20]. Owing to short delivery cycles, high prices, as well as high inventory holding and penalty costs, components must be manufactured in a production sharing model by manufacturers within a supply chain. Although the inventory holding cost increases if the throughput is too high or the production is too early, the penalty cost might occur if the throughput is too low or the 
production is too late. Therefore, production planning is especially important when determining production quantity and production time. In practice, owing to uncertain yield of machines in mobile phone production, the fuzzy theory is applicable in assisting managers to obtain solutions.

Pioneered by Zadeh [21], the fuzzy set theory was applied by Bellman and Zadeh [22] to solve decisionmaking problems related to uncertain characteristics. Zimmermann [23] first applied the fuzzy set theory to conventional Linear Programming (LP) models. This model considers LP problems with a fuzzy goal and fuzzy constraints. Multidisciplinary studies have extended the applicability of Fuzzy Multi-Objective Linear Programming (FMOLP) to production-planning problems. By applying an FMOLP model, Peidro and Vasant [24] attempted to solve a master planning problem for a centralized replenishment, production, and distribution ceramic tile supply chain. That model examines the feasibility of maximizing the total gross margin, minimizing backorder quantities, and minimizing idle production time in a multi-item and multiperiod logistic environment. Ganesan et al. [25] developed a hybrid neuro-swarm optimization approach to solve the economic dispatch problem in power generation systems. Jiménez et al. [26] developed a multi-objective evolutionary method for solving a fuzzy nonlinear optimization problem in production planning for the manufacture of chocolate. Vasant [27] developed hybrid line search that simulated annealing and pattern search methods for solving a large-scale non-linear fuzzy programming problem in industrial production planning.

In sum, despite the considerable attention paid to production planning in mobile phone manufacturing, how cost-effectiveness analysis exactly affects the decision-making process has seldom been addressed.

\section{Model formulation}

\subsection{Problem description}

This work considers lot-sizing production planning decision problems with respect to mobile phone manufacturing systems (Figure 1). Mobile phone manufacturing consists of the design, manufacturing, and assembly/testing stages. To successfully develop a core technology, a mobile phone factory must be proficient in the design stage and outsource other noncore operations. The design stage includes research and design of the new product. The manufacturing stage includes manufacturing contract (e.g., electronics components, electromechanical components, mechanics components, accessory, and batteries). Finally, the assembly and testing stage includes sub-assembly, PCB assembly, box assembly, full product assembly, and testing.

\subsection{DM processes in mobile phone manufacturing}

This section concerns the mobile phone manufacturing sector problem. In this section, production managers must consider the mobile phone manufacturing sector problem. The mobile phone manufacturing industry faces more complex imprecise/fuzzy problems when outsourcing operations. In production planning decision problems involving mobile phone manufacturing systems, input data or parameters (e.g., forecasting demand, resources, costs, and objective function) are often imprecise, resulting in a fuzzy objective function. For instance, the objective function of total annual manufactured costs may be $\mathrm{NT} \$ 7$ million, annual holding costs may be NT $\$ 0.3$ million, and annual penalty costs may be NT $\$ 0.1$ million. This imprecision warrants the need for a set of fuzzy multi-objective models to produce a set of compromising solutions. Therefore, the optimal lot-size for the manufacturing stage is determined using a lot-sizing production planning decision problem. Figure 2 presents the flow chart of the DM process.

\subsection{Notations}

\section{Set of indices}

$i \quad$ Number of components: $i=1,2, \ldots, I$;

$j \quad$ Number of vendors: $j=1,2, \ldots, J$;

$t \quad$ Number of periods: $t=0,1,2, \ldots, T$, where $t=0$ denotes the due date and $t=T$ denotes total number of periods in the time horizon.

\section{Parameters}

$f c_{i t} \quad$ Fixed cost allocated for the $i$ th components in period $t$ ( $\$ /$ time);

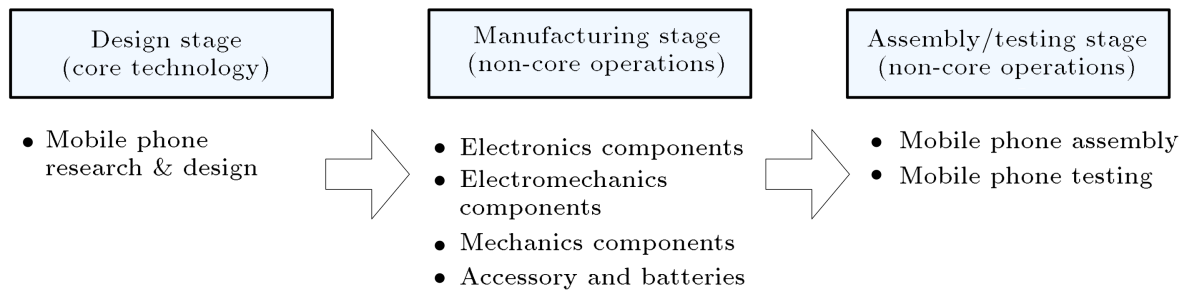

Figure 1. Mobile phone manufacturing flow. 


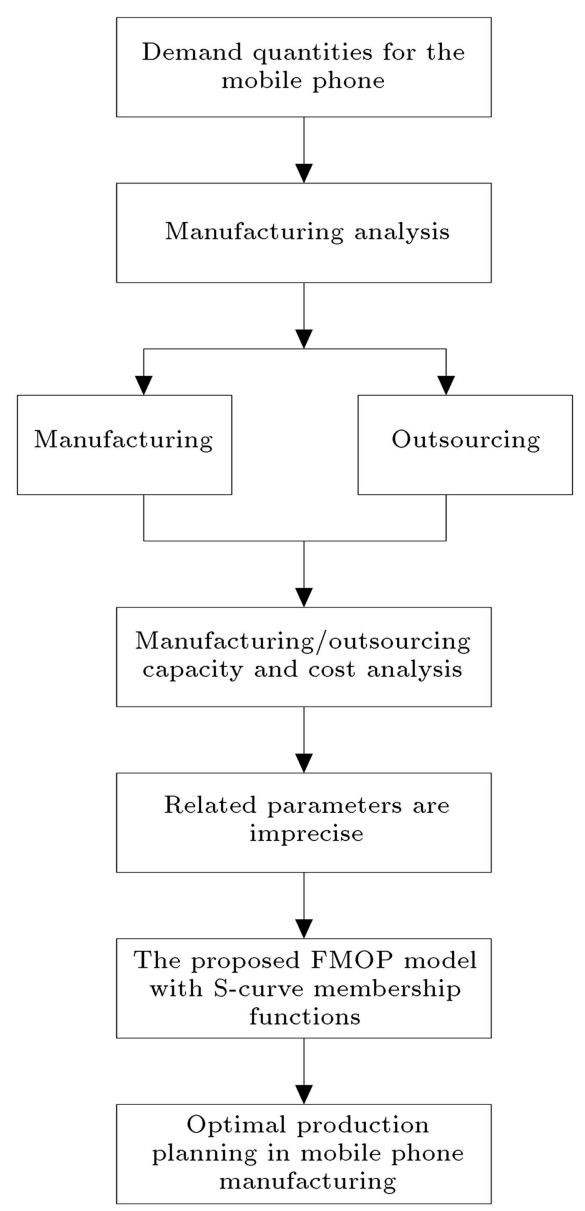

Figure 2. Block diagram of the DM process.

$m c_{i t} \quad$ Manufacturing cost per unit for the $i$ th components in period $t(\$ /$ unit);

$v c_{i j t} \quad$ Purchasing cost per unit of the $j$ th vendor for the $i$ th components in period $t$ (\$/unit);

$i c_{i} \quad$ Inventory holding cost per unit of the $i$ th components (\$/unit);

$D_{i} \quad$ Demand quantities for the $i$ th components (units);

$\theta_{i t} \quad$ Yield parameter for the $i$ th components in period $t, 0 \leq \theta_{i t} \leq 1$;

$y_{i j t} \quad$ Yield parameter of the $j$ th vendor for the $i$ th components in period $t$, $0 \leq y_{i j t} \leq 1(\%)$

$p c_{i} \quad$ Penalty cost per unit of the $i$ th components (\$/unit);

$M L_{i t} \quad$ Minimum manufacturing availability for the $i$ th components in period $t$ (units);

$M U_{i t} \quad$ Maximum manufacturing availability for the $i$ th components in period $t$ (units);
$V M_{i j t}^{\min } \quad$ Minimum supply availability of the $j$ th vendor for the $i$ th components in period $t$ (units);

$V M_{i j t}^{\max } \quad$ Maximum supply availability of the $j$ th vendor for the $i$ th components in period $t$ (units);

$s p_{i} \quad$ Holding space per unit of the $i$ th components ( $\mathrm{m}^{2} /$ unit);

$I_{i}^{\max } \quad$ Maximum holding space of $i$ th components $\left(\mathrm{m}^{2}\right)$.

Decision variables

$m q_{i t} \quad$ Lot-sizing of manufacturing for the $i$ th components in period $t$ (units);

$v q_{i j t} \quad$ Lot-sizing of outsourcing of the $j$ th vendor for the $i$ th components in period $t$ (units).

\subsection{Fuzzy Multi-Objective Programming (FMOP) model}

In practice, most production-planning decision problems in mobile phone manufacturing are concerned with total manufacturing costs. To reduce production costs and, consequently, improve the competitiveness of mobile phone manufacturers, most productionplanning decision problems in this field that can be found in the literature involve minimization of total manufacturing costs $[5,6,12,18]$.

To minimize total manufacturing cost, decision models must account for both inventory holding and penalty costs. These objectives are typically fuzzy because information is incomplete and/or unavailable over the middle planning horizon. Accordingly, the following three objective functions are simultaneously considered in the development of the proposed FMOP model:

\section{- Objective functions}

1. Minimizing total manufacturing cost: The total manufacturing cost includes production cost, outsourcing cost, inventory holding cost, and penalty cost:

$$
\begin{aligned}
\operatorname{Min} Z_{1} \cong \sum_{i=1}^{I} \sum_{t=1}^{T}\left[f c_{i t}+\left(m c_{i t} \cdot m q_{i t}\right)\right] \\
+\sum_{i=1}^{I} \sum_{j=1}^{J} \sum_{t=1}^{T}\left[d c_{i j} \cdot v q_{i j t}\right] \\
+\sum_{i=1}^{I} \sum_{j=1}^{J} \sum_{t=1}^{T}\left[v c_{i j t} \cdot v q_{i j t}\right] \\
+\sum_{i=1}^{I} \sum_{j=1}^{J} \sum_{t=1}^{T}\left[(t-1) \cdot i c_{i} \cdot\left(m q_{i t}+v q_{i j t}\right)\right]
\end{aligned}
$$




$$
\begin{aligned}
& +\sum_{i=1}^{I} \sum_{t=1}^{T}\left[D_{i}-\left(m q_{i t} \cdot \theta_{i t}\right.\right. \\
& \left.\left.+\left(\sum_{j=1}^{J} v q_{i j t} \cdot y_{i j t}\right)\right)\right] \cdot p c_{i} .
\end{aligned}
$$

2. Minimizing inventory holding costs.

$$
\begin{gathered}
\operatorname{Min} Z_{2} \cong \sum_{i=1}^{I} \sum_{t=1}^{T} \sum_{t=1}^{T}[(t-1) \\
\left.. i c_{i} .\left(m q_{i t}+v q_{i j t}\right)\right] .
\end{gathered}
$$

3. Minimizing penalty costs.

$$
\begin{aligned}
& \text { Min } Z_{3} \cong \sum_{i=1}^{I} \sum_{t=1}^{T}\left[D_{i}-\left(m q_{i t} . \theta_{i t}\right.\right. \\
&\left.\left.+\left(\sum_{j=1}^{J} v q_{i j t} . y_{i j t}\right)\right)\right] \cdot p c_{i} .
\end{aligned}
$$

As the fuzzified version of "=", the symbol

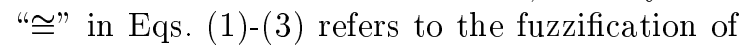
aspiration levels. For each objective function of the proposed FMOP model, the DM is assumed to have a fuzzy objective. For instance, for total annual manufactured costs, the objective may be $\$ 1$ million; for annual total inventory holding costs, the objective may be $\$ 0.2$ million; for annual total penalty costs, the objective may be $\$ 0.1$ million. Hence, Eqs. (1)-(3) are fuzzy and have imprecise aspiration levels. They also incorporate variations of the DM judgments with respect to solutions for the fuzzy optimization problem. These fuzzy goals require simultaneous optimization by a DM in the framework of fuzzy aspiration levels.

- Constraints

1. Manufacturing capacity:

$$
\begin{aligned}
& \sum_{i=1}^{I} \sum_{t=1}^{T} m q_{i t} \leq m q_{i t}^{\max } \quad \forall i, t, \\
& m q_{i t} \leq M U_{i t} \quad \forall i, t, \\
& m q_{i t} \geq M L_{i t} \quad \forall i, t .
\end{aligned}
$$

2. Vendor capacity:

$$
\begin{aligned}
& v q_{i j t} \leq V M_{i j t}^{\max } \quad \forall i, j, t, \\
& v q_{i j t} \geq V M_{i j t}^{\min } \quad \forall i, j, t .
\end{aligned}
$$

3. Warehouse space:

$$
\sum_{i=1}^{I} \sum_{t=1}^{T}\left(m q_{i t}+\sum_{j=1}^{J} v q_{i j t}\right) \cdot s p_{i} \leq I_{i t}^{\max } \quad \forall i, j, t .
$$

4. Non-negativity:

$$
m q_{i t}, v q_{i j t} \geq 0 \quad \forall i, j, t .
$$

\subsection{Modified S-curve membership function}

Many membership functions are available, including linear, piecewise linear, and nonlinear ones, with the nonlinear membership function being more flexible than general linear ones. More flexible and practical production plans are thus necessary because the industrial features of smart phones differ from those of other industries. Therefore, this work attempts to explain uncertain or fuzzy features in practice by using the modified S-curve membership function.

The original FMOP model for solving previous problems is applicable to the modified S-curve membership function developed by Vasant et al. [11]. While sufficiently flexible to describe the imprecision in fuzzy parameters, the modified S-curve membership function is a unique case of logistic function in which the specific values are $B, C$, and $\alpha$. These values must be derived. This logistic function, as given by Eq. (11) and described in Figure 3, is exhibited as an S-shaped membership. Thus, a modified S-curve membership function is as follows [11]:

$$
\mu(x)= \begin{cases}1 & x<x^{a} \\ 0.999 & x=x^{a} \\ \frac{B}{1+C e^{\alpha x}} & x^{a}<x<x^{b} \\ 0.001 & x=x^{b} \\ 0 & x>x^{b}\end{cases}
$$

where $\mu$ denotes the degree of membership function. Eq. (11) is the membership function and it is defined as $0.001 \leq \mu(x) \leq 0.999$. This range is selected because in mobile phone manufacturing, the availability capacity

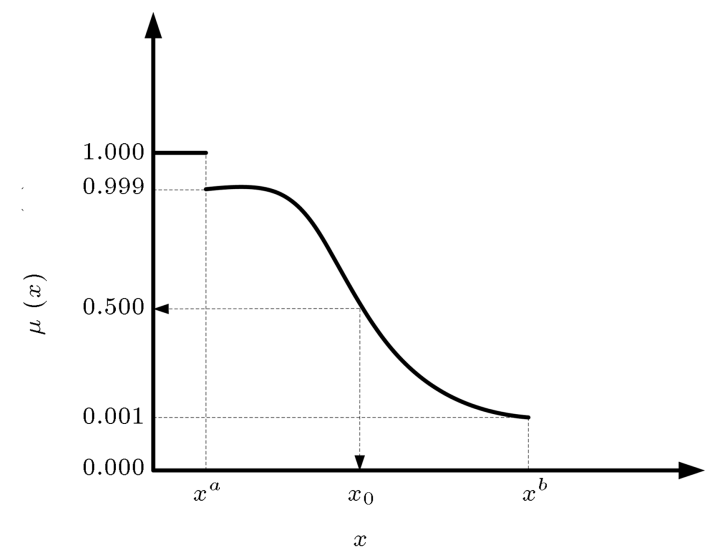

Figure 3. S-curve membership function. 
and warehouse space do not always need to satisfy $100 \%$ of the requirements. The overall manufacturing, inventory holding, and penalty costs are not simultaneously $0 \%$. Thus, there is a range between $x^{a}$ and $x^{b}$ with $0.001 \leq \mu(x) \leq 0.999$. This concept of range of $\mu(x)$ is used to solve real-world production planning problems in mobile phone manufacturing.

To obtain the value of $B, C$ and $\alpha$ are used to readjust the $x$-axis as $x^{a}=0$ and $x^{b}=1$. Nowakowska [28] has completed such a readjusting experiment in social sciences. The value for $B$ is 1 , for $C$ is 0.001001001 , and for $\alpha$ is 13.81350956 .

\subsection{Solving the FMOP model problem}

In Eq. (11), the modified S-curve membership function by Vasant et al. [1] represents the fuzzy set corresponding to each objective:

$$
f_{g}\left(Z_{g}\right)=\left\{\begin{array}{lc}
1 & Z_{g}<Z_{g}^{l} \\
0.999 & Z_{g}=Z_{g}^{l} \\
\frac{B}{1+C e^{\alpha\left(z_{g}-z_{g}^{l} / z_{g}^{u}-z_{g}^{l}\right)}} & z_{g}^{l}<z_{g}<z_{g}^{u} \\
0.001 & z_{g}=z_{g}^{u} \\
0 & z_{g}>z_{g}^{u}
\end{array}\right.
$$

$$
g=1,2, \ldots, G
$$

where $z_{g}^{u}$ and $z_{g}^{l}$ denote the upper and lower bounds of value domains for the fuzzy objective function, respectively, for the $g$ th objective function, $z_{g}$. Each membership function can be determined by requiring the DM to specify the imprecise objective value interval $\left[z_{g}^{l}, z_{g}^{u}\right]$

Several methods have been extensively adopted to solve FMOP model problems. Torabi and Hassini [29] developed a fuzzy programming method as a combination of the previous methods of Lai and Hwang [30] and Selim and Ozkarahan [31]. This work adopts the original FMOP model for solving the production planning problem used by Torabi and Hassini [29]. According to Torabi and Hassini [29], an FMOP model can be transformed into an equivalent conventional single-objective model as follows:

$$
\begin{aligned}
& \operatorname{Max} \lambda\left(z_{g}\right)=\gamma \cdot \lambda_{0}+(1-\gamma) \cdot \sum_{g=1}^{G} w_{g} \cdot f_{g}\left(z_{g}\right), \\
& \text { s.t. } \quad \lambda_{0} \leq f_{g}\left(z_{g}\right), \quad g=1,2, \ldots, G, \\
& z_{g} \in F\left(z_{g}\right), \\
& \gamma, \lambda_{0} \in[0,1],
\end{aligned}
$$

where $f_{g}\left(z_{g}\right)$ and $\lambda_{0}=\min \left\{f_{g}\left(z_{g}\right)\right\}$ denote the satisfaction degree of the $g$ th objective function and the minimum satisfaction degree of the objectives, respectively; $w_{g}$ represents the weight coefficient that describes the relative importance among the $g$ th fuzzy objectives $0<w_{g} \leq 1$; and $\gamma$ refers to the compensation coefficient that presents the minimum satisfaction degree of the objectives according to the DM's preferences by regulating the value of parameter $\gamma[29]$.

Following the fuzzy decision-making concept of Bellman and Zadeh [22] and the fuzzy programming method of Torabi and Hassini [29], the proposed FMOP model can be transformed into an equivalent singleobjective non-linear programming model for solving a production planning problem in mobile phone manufacturing in Eqs. (4)-(10) and the following relations:

$$
\begin{aligned}
& \operatorname{Max} \gamma \cdot \lambda_{0}+(1-\gamma) \cdot\left[w_{1} \cdot f_{1}\left(z_{1}\right)+w_{2} \cdot f_{2}\left(z_{2}\right)\right. \\
& \left.+w_{3} \cdot f_{3}\left(z_{3}\right)\right] \\
& \text { s.t. } \\
& \lambda_{0} \leq f_{1}\left(z_{1}\right) \\
& \lambda_{0} \leq f_{2}\left(z_{2}\right) \\
& \lambda_{0} \leq f_{3}\left(z_{3}\right) \\
& \gamma, \lambda_{0} \in[0,1] .
\end{aligned}
$$

This work presents an interactive solution procedure of an FMOP model for the production planning problem of mobile phone manufacturing with modified Scurve membership functions indicating fuzzy objectives. Based on the fuzzy programming solution method of Torabi and Hassini [29], the proposed procedure obtains a single-objective crisp non-linear programming model. Thus, the interactive solution procedure of an FMOP model is implemented as follows:

Step 1. Construct an original FMOP model for solving the production planning problem in mobile phone manufacturing with multiple fuzzy objectives based on Eqs. (1)-(10);

Step 2. Evaluate each fuzzy objective function based on the knowledge of DMs or experts, $z_{g}$, which corresponds to the modified S-curve membership functions $f_{g}\left(z_{g}\right)$;

Step 3. Derive the corresponding relative importance of the objective functions $\left(w_{g}\right)$ and coefficient of compensation $(\gamma)$;

Step 4. Use a minimum operator to integrate the fuzzy set in order to transform the original FMOP model into an equivalent single-objective model;

Step 5. Solve the single-objective crisp non-linear programming problem to obtain the initial compromise solution; and 
Table 1. Relevant components data.

\begin{tabular}{ccccccc}
\hline Components & $\boldsymbol{i}=\mathbf{1}$ & $\boldsymbol{i}=\mathbf{2}$ & $\boldsymbol{i}=\mathbf{3}$ & $\boldsymbol{i}=\mathbf{4}$ & $\boldsymbol{i}=\mathbf{5}$ & $\boldsymbol{i}=\mathbf{6}$ \\
\hline$i c_{\boldsymbol{i}}(\$ /$ unit-period $)$ & 2.7 & 2.3 & 1.0 & 2.3 & 0.1 & 0.2 \\
$p c_{\boldsymbol{i}}$ (\$/unit) & 50 & 44 & 25 & 48 & 4 & 9 \\
$s p_{i}\left(\mathrm{~m}^{2} /\right.$ unit $)$ & 0.03 & 0.10 & 0.10 & 0.08 & 0.10 & 0.05 \\
$D_{i}$ (units) & 25000 & 26000 & 28000 & 25000 & 20000 & 22000 \\
$I_{i t}^{\max }\left(\mathrm{m}^{2}\right)$ & 2700 & 3700 & 4200 & 3000 & 1800 & 1850 \\
\hline
\end{tabular}

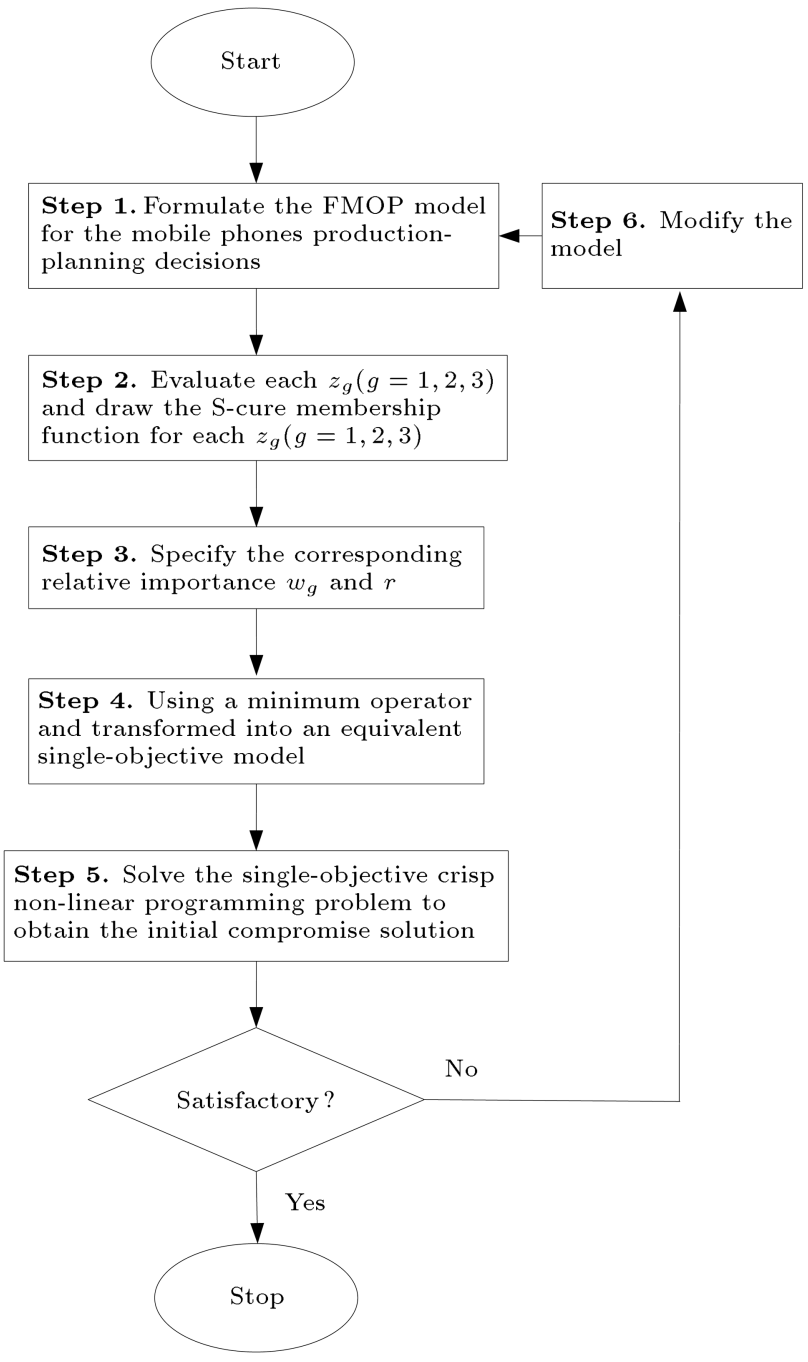

Figure 4. Block diagram of interactive FMOP model development.

Step 6. Complete and modify the interactive model until the measurements of each fuzzy objective function and coefficients $\left(w_{g}, \gamma\right)$ are satisfactory.

Figure 4 shows a block diagram of the interactive solution procedure for the development of an FMOP model.

\section{Computational experiments}

This section evaluates the accuracy and performance of the proposed model to achieve efficient production planning in mobile phone manufacturing by using a real-world test case in Taiwan. In all of the experiments herein, a personal computer equipped with an Intel ${ }^{\circledR}$ 3.0GHZ CPU and 504 MB RAM is used.

\subsection{Test cases}

$\mathrm{H}$ Company functions as the test object in this work. The main product of $\mathrm{H}$ Company is smart phones, while its subsidiaries produce the main components for smart phones including key chips $(i=1)$, panels $(i=2)$, circuit boards $(i=3)$, electronic parts $(i=4)$, cases $(i=5)$, and cells $(i=6)$. Each component has three vendors $(j=1,2,3)$. The production plan is based on one quarter to be executed in the four quarters $(t=1,2,3,4)$ of a year. Table 1 summarizes the part parameters data including inventory holding costs, penalty costs, part volume, demand, and storage capacity. Table 2 summarizes the homemaking production data including fixed costs, homemaking costs, and capacity constraint. Table 3 displays the vendor production data including the production costs associated with the vendor, capacity constraint, and production yield.

\subsection{Solution procedure for mobile phone manufacturing}

The solution procedure defined in Section 3.4 is followed. Based on a fuzzy multi-objective model, this work evaluates the feasibility of the proposed model. The interactive solution procedure with the proposed FMOP model to solve the production planning problem for the case of $\mathrm{H}$ Company is described as follows.

The original FMOP model is first formulated to solve the multi-component, multi-supplier, and multi-time-period production planning problems using Eqs. (1)-(10). The original FMOP model for the mobile phone manufacturing case is then derived by using the conventionally adopted single-objective nonlinear programming model to obtain initial solutions for each objective function. As is assumed here, a DM specifies the corresponding possible value interval for each fuzzy objective, as the precise value can be determined based on the experience of DMs; the equal membership group of the decision makers is normally in an interval $[0,1]$. The mathematical programming software program LINGO version 10.0 is used to solve real world production plan- 
Table 2. Relevant production data.

\begin{tabular}{|c|c|c|c|c|c|c|c|}
\hline \multicolumn{2}{|c|}{ Components } & \multirow{2}{*}{$\begin{array}{l}\boldsymbol{i}=\mathbf{1} \\
58000\end{array}$} & \multirow{2}{*}{$\begin{array}{l}\boldsymbol{i}=\mathbf{2} \\
56000\end{array}$} & \multirow{2}{*}{$\begin{array}{l}\boldsymbol{i}=\mathbf{3} \\
48000\end{array}$} & \multirow{2}{*}{$\frac{i=4}{56000}$} & \multirow{2}{*}{$\frac{\boldsymbol{i}=\mathbf{5}}{48000}$} & \multirow{2}{*}{$\begin{array}{l}\boldsymbol{i}=\mathbf{6} \\
49000\end{array}$} \\
\hline \multirow{4}{*}{$t=1$} & $f c_{i t}$ & & & & & & \\
\hline & $m c_{i t}$ & 37 & 28 & 16 & 32 & 2 & 6 \\
\hline & $M U_{i t}$ & 2600 & 2400 & 2300 & 2500 & 2200 & 2100 \\
\hline & $M L_{i t}$ & 1000 & 1000 & 1000 & 1000 & 1000 & 1000 \\
\hline \multirow{4}{*}{$t=2$} & $f c_{i t}$ & 58000 & 56000 & 48000 & 56000 & 48000 & 49000 \\
\hline & $m c_{i t}$ & 36 & 27 & 15 & 31 & 2 & 6 \\
\hline & $M U_{i t}$ & 2600 & 2400 & 2300 & 2500 & 2200 & 2100 \\
\hline & $M L_{i t}$ & 1000 & 1000 & 1000 & 1000 & 1000 & 1000 \\
\hline \multirow{4}{*}{$t=3$} & $f c_{i t}$ & 58000 & 56000 & 48000 & 56000 & 48000 & 49000 \\
\hline & $m c_{i t}$ & 32 & 26 & 14 & 30 & 2 & 6 \\
\hline & $M U_{i t}$ & 2600 & 2400 & 2300 & 2500 & 2200 & 2100 \\
\hline & $M L_{i t}$ & 1000 & 1000 & 1000 & 1000 & 1000 & 1000 \\
\hline \multirow{4}{*}{$t=4$} & $f c_{i t}$ & 58000 & 56000 & 48000 & 56000 & 48000 & 49000 \\
\hline & $m c_{i t}$ & 31 & 25 & 13 & 29 & 2 & 6 \\
\hline & $M U_{i t}$ & 2600 & 2400 & 2300 & 2500 & 2200 & 2100 \\
\hline & $M L_{i t}$ & 1000 & 1000 & 1000 & 1000 & 1000 & 1000 \\
\hline \multicolumn{2}{|c|}{$\theta_{i t}$} & 0.90 & 0.89 & 0.91 & 0.90 & 0.95 & 0.94 \\
\hline
\end{tabular}

Table 3. Relevant vendor data.

\begin{tabular}{|c|c|c|c|c|c|c|c|c|c|c|c|c|c|c|c|c|c|c|c|}
\hline \multirow{2}{*}{\multicolumn{2}{|c|}{$\frac{\text { Components }}{j}$}} & \multicolumn{3}{|c|}{$i=1$} & \multicolumn{3}{|c|}{$i=2$} & \multicolumn{3}{|c|}{$i=3$} & \multicolumn{3}{|c|}{$i=4$} & \multicolumn{3}{|c|}{$i=5$} & \multicolumn{3}{|c|}{$i=6$} \\
\hline & & 1 & 2 & 3 & 1 & 2 & 3 & 1 & 2 & 3 & 1 & 2 & 3 & 1 & 2 & 3 & 1 & 2 & 3 \\
\hline & $v c_{i j t}$ & 37 & 38 & 39 & 29 & 27 & 27 & 14 & 17 & 18 & 33 & 31 & 32 & 3 & 4 & 3 & 7 & 8 & 5 \\
\hline \multirow[t]{3}{*}{$t=1$} & $V M_{i j t}^{\max }$ & 2600 & 2600 & 2600 & 2400 & 2400 & 2400 & 2300 & 2300 & 2300 & 2500 & 2500 & 2500 & 2200 & 2200 & 2200 & 2100 & 2100 & 2100 \\
\hline & $V M_{i j t}^{\min }$ & 500 & 500 & 500 & 500 & 500 & 500 & 500 & 500 & 500 & 500 & 500 & 500 & 500 & 500 & 500 & 500 & 500 & 500 \\
\hline & $v c_{i j t}$ & 36 & 37 & 38 & 28 & 26 & 26 & 14 & 15 & 16 & 32 & 30 & 31 & 3 & 4 & 3 & 7 & 8 & 5 \\
\hline \multirow[t]{3}{*}{$t=2$} & $V M_{i j t}^{\max }$ & 2600 & 2600 & 2600 & 2400 & 2400 & 2400 & 2300 & 2300 & 2300 & 2500 & 2500 & 2500 & 2200 & 2200 & 2200 & 2100 & 2100 & 2100 \\
\hline & $V M_{i j t}^{\min }$ & 500 & 500 & 500 & 500 & 500 & 500 & 500 & 500 & 500 & 500 & 500 & 500 & 500 & 500 & 500 & 500 & 500 & 500 \\
\hline & $v c_{i j t}$ & 35 & 36 & 37 & 28 & 26 & 26 & 14 & 15 & 16 & 32 & 30 & 31 & 3 & 4 & 3 & 7 & 8 & 5 \\
\hline \multirow[t]{3}{*}{$t=3$} & $V M_{i j t}^{\max }$ & 2600 & 2600 & 2600 & 2400 & 2400 & 2400 & 2300 & 2300 & 2300 & 2500 & 2500 & 2500 & 2200 & 2200 & 2200 & 2100 & 2100 & 2100 \\
\hline & $V M_{i j t}^{\min }$ & 500 & 500 & 500 & 500 & 500 & 500 & 500 & 500 & 500 & 500 & 500 & 500 & 500 & 500 & 500 & 500 & 500 & 500 \\
\hline & $v c_{i j t}$ & 34 & 35 & 35 & 27 & 25 & 27 & 13 & 14 & 14 & 31 & 29 & 30 & 3 & 4 & 3 & 7 & 8 & 5 \\
\hline \multirow[t]{3}{*}{$t=4$} & $V M_{i j t}^{\max }$ & 2600 & 2600 & 2600 & 2400 & 2400 & 2400 & 2300 & 2300 & 2300 & 2500 & 2500 & 2500 & 2200 & 2200 & 2200 & 2100 & 2100 & 2100 \\
\hline & $V M_{i j t}^{\min }$ & 500 & 500 & 500 & 500 & 500 & 500 & 500 & 500 & 500 & 500 & 500 & 500 & 500 & 500 & 500 & 500 & 500 & 500 \\
\hline & $y_{i j t}$ & 0.92 & 0.91 & 0.92 & 0.91 & 0.93 & 0.91 & 0.93 & 0.92 & 0.93 & 0.92 & 0.94 & 0.92 & 0.94 & 0.95 & 0.94 & 0.95 & 0.96 & 0.95 \\
\hline
\end{tabular}

ning problems in mobile phone manufacturing. Additionally, the crisp single-objective non-linear programming model is derived for a production planning problem in mobile phone manufacturing. Table 4 summarizes the initial results obtained by the single-objective non-linear programming model and the corresponding interval values for each fuzzy objective.

Modified S-curve membership functions for the approach of Vasant et al. [11] are described. Imprecise production planning from mobile phone manufacturing can be formulated via Eq. (11), as follows: 
Table 4. Preliminary solutions and interval values for each objective function.

\begin{tabular}{ccccc}
\hline Item & LP-1 & LP-2 & LP-3 & $\left(z_{g}^{l}, z_{g}^{u}\right)$ \\
\hline Objective function & Min $z_{1}$ & Min $z_{2}$ & Min $z_{3}$ & - \\
Satisfactory degree $(\%)$ & $100 \%$ & $100 \%$ & $100 \%$ & - \\
$z_{1}(\$)$ & $4,153,672^{*}$ & $4,278,647$ & $4,398,375$ & $(4,000,000,6,000,000)$ \\
$z_{2}(\$)$ & 275,066 & $214,202^{*}$ & 355,759 & $(200,000,400,000)$ \\
$z_{3}(\$)$ & 20,163 & 382,745 & $0^{*}$ & $(0,400,000)$ \\
\hline
\end{tabular}

*The optimal value with the ordinary single-objective non-linear programming model.

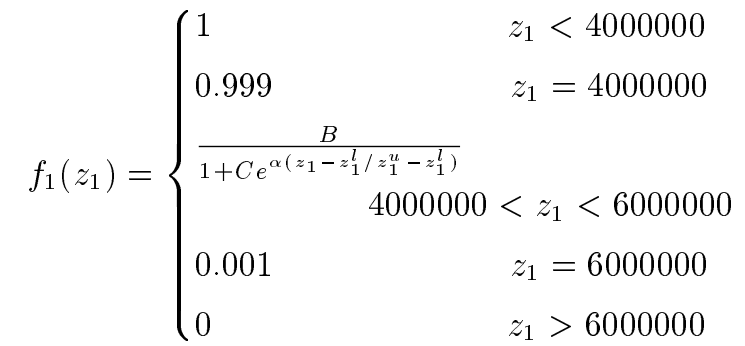

$$
\begin{aligned}
& f_{2}\left(z_{2}\right)=\left\{\begin{array}{lrl}
1 & z_{2}<200000 \\
0.999 & z_{2}=200000 \\
\frac{B}{1+C e^{\alpha\left(z_{2}-z_{2}^{l} / z_{2}^{u}-z_{2}^{l}\right)}} & \\
0.001 & 200000 & <z_{2}<400000 \\
0 & z_{2}=400000 \\
0 & z_{2}>400000
\end{array}\right. \\
& f_{3}\left(z_{3}\right)= \begin{cases}1 & z_{3}<0 \\
0.999 & z_{3}=0 \\
\frac{B}{1+C e^{\alpha\left(z_{3}-z_{3}^{l} / z_{3}^{u}-z_{3}^{l}\right)}} & 0<z_{3}<400000 \\
0.001 & z_{3}=400000 \\
0 & z_{3}>400000\end{cases}
\end{aligned}
$$

All of the modified S-curve membership functions adopt the same values for $B, C$, and $\alpha$ parameters. These values are $B=1, C=0.001001001$, and $\alpha=13.813$. The coefficient of compensation is $\gamma=0.1$. The DM linguistically provides the relative importance of the objectives as $w_{1}>w_{2}=w_{3}$, meaning that for the DM, minimizing total manufacturing cost is more important than minimizing inventory holding cost or penalty cost. The corresponding weights $\left(w_{1}, w_{2}, w_{3}\right)=(0.4,0.3,0.3)$ for the three fuzzy objectives are obtained with reference to historical data.

The proposed model is implemented to minimize total manufacturing, inventory holding, and penalty costs, simultaneously, yielding $z_{1}=\mathrm{NT} \$ 4,191,614, z_{2}$ $=\mathrm{NT} \$ 253,742$, and $z_{3}=\mathrm{NT} \$ 38,254$, respectively. Additionally, DM adjusts $\left(z_{g}^{l}, z_{g}^{u}\right)$ for each fuzzy objective to obtain the satisfactory degree of $87.54 \%$. Table 5 summarizes the optimal production planning in mobile phone manufacturing.

\subsection{Sensitivity analysis}

Exactly how the variation for compensation coefficient $\gamma$ affects the degree of satisfaction is analyzed, as well as how it affects $z_{1}, z_{2}$, and $z_{3}$. Five runs are set from small to large for the compensation coefficient, $\gamma: 0.1,0.3,0.5,0.7$, and 0.9 , respectively, while 0.1 is the setting of the initial compensation coefficient, $\gamma$ (Table 6).

Sensitivity analysis results indicate that the variable compensation coefficient for the overall manufacturing, inventory, and penalty costs affect the objective functions $\left(z_{1}, z_{2}, z_{3}\right)$ and degree of satisfaction (Figures 5-7). The increasing compensation coefficient, $\gamma$, controls not only the minimum satisfaction level of the objectives but also, implicitly, the degree of compromise among the objectives. The degree of satisfaction gradually declines from $87.54 \%$ to $84.26 \%$. The proposed formulation can be used to obtain both unbalanced and balanced compromised solutions for a given problem, according to the DM's preferences, by adjusting the parameter $\gamma$ [29]. A low $\gamma$ value means that the model emphasizes the need for greater satisfaction in relation to the most heavily weighted objectives and deemphasizes the need for a higher overall degree of DM satisfaction. Table 6 reveals that $\gamma=0.1$ yields the lowest inventory holding

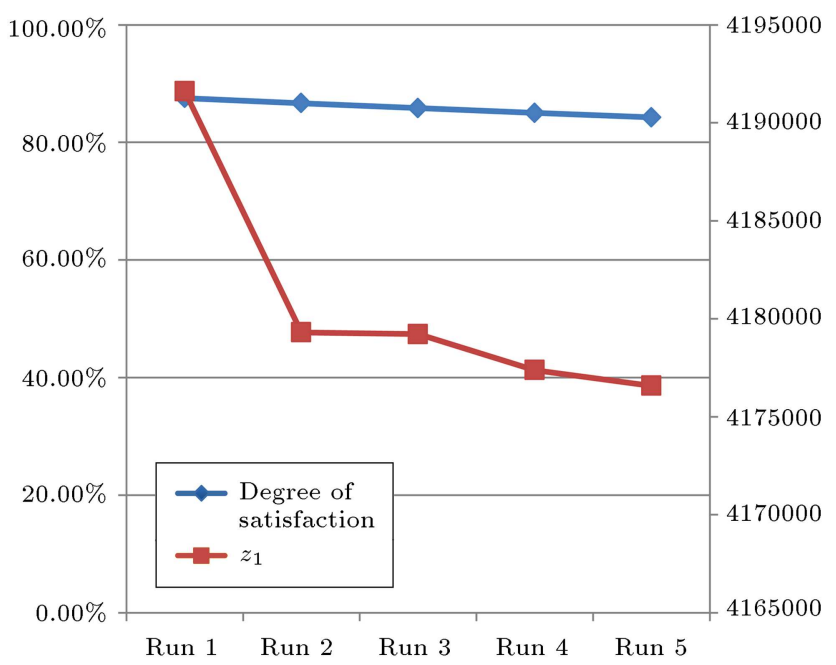

Figure 5. Overall manufacturing costs and degree of satisfaction for various $\gamma$ values. 
Table 5. Optimal production planning in mobile phone manufacturing.

\begin{tabular}{|c|c|c|c|c|c|c|}
\hline \multicolumn{2}{|c|}{ Components demand } & \multirow{2}{*}{$\frac{\text { Make }}{2600}$} & \multirow{2}{*}{$\begin{array}{c}\boldsymbol{j}=\mathbf{1} \\
2600\end{array}$} & \multirow{2}{*}{$\begin{array}{c}\boldsymbol{j}=\mathbf{2} \\
2600\end{array}$} & \multirow{2}{*}{$\frac{\boldsymbol{j}=\mathbf{3}}{2600}$} & \multirow[t]{2}{*}{ Shortage quantity } \\
\hline \multirow{4}{*}{$D_{1}=25000$} & $t=1$ & & & & & \\
\hline & $t=2$ & 2600 & 2500 & 2500 & 2500 & \multirow{3}{*}{0} \\
\hline & $t=3$ & 2600 & 2400 & 930 & 0 & \\
\hline & $t=4$ & 1000 & 0 & 0 & 0 & \\
\hline \multirow{4}{*}{$D_{2}=25000$} & $t=1$ & 2400 & 2400 & 2400 & 2400 & \multirow{4}{*}{0} \\
\hline & $t=2$ & 2055 & 2300 & 2300 & 2300 & \\
\hline & $t=3$ & 2400 & 0 & 2200 & 2200 & \\
\hline & $t=4$ & 1000 & 0 & 1114 & 0 & \\
\hline \multirow{4}{*}{$D_{3}=25000$} & $t=1$ & 1000 & 2300 & 0 & 0 & \multirow{4}{*}{0} \\
\hline & $t=2$ & 2300 & 2200 & 2200 & 0 & \\
\hline & $t=3$ & 2300 & 2100 & 2100 & 2100 & \\
\hline & $t=4$ & 2300 & 2000 & 2000 & 2000 & \\
\hline \multirow{4}{*}{$D_{4}=25000$} & $t=1$ & 2500 & 2500 & 2500 & 2500 & \multirow{4}{*}{0} \\
\hline & $t=2$ & 2500 & 2400 & 2400 & 2400 & \\
\hline & $t=3$ & 2500 & 0 & 2300 & 1703 & \\
\hline & $t=4$ & 1000 & 0 & 0 & 0 & \\
\hline \multirow{4}{*}{$D_{5}=28000$} & $t=1$ & 1000 & 0 & 0 & 0 & \multirow{4}{*}{2955} \\
\hline & $t=2$ & 2200 & 2100 & 885 & 2100 & \\
\hline & $t=3$ & 2200 & 2000 & 2000 & 2000 & \\
\hline & $t=4$ & 2200 & 1900 & 1900 & 1900 & \\
\hline \multirow{4}{*}{$D_{6}=28000$} & $t=1$ & 1860 & 0 & 0 & 2100 & \multirow{4}{*}{1953} \\
\hline & $t=2$ & 2100 & 2000 & 0 & 2000 & \\
\hline & $t=3$ & 2100 & 1900 & 1900 & 1900 & \\
\hline & $t=4$ & 2100 & 1800 & 1800 & 1800 & \\
\hline$z_{1}$ & \multicolumn{6}{|c|}{$\$ 4,191,614$} \\
\hline$z_{2}$ & \multicolumn{6}{|c|}{$\$ 253,742$} \\
\hline$z_{3}$ & \multicolumn{6}{|c|}{$\$ 38,254$} \\
\hline Degree of satisfac & \multicolumn{6}{|c|}{$87.54 \%$} \\
\hline
\end{tabular}

Table 6. Sensitivity analysis of compensation coefficient variations.

\begin{tabular}{cccccc}
\hline Items & Run 1 (original) & Run 2 & Run 3 & Run 4 & Run 5 \\
\hline$\gamma$ & 0.1 & 0.3 & 0.5 & 0.7 & 0.9 \\
$z_{1}(\$)$ & $4,191,614$ & $4,179,305$ & $4,179,215$ & $4,177,384$ & $4,176,584$ \\
$z_{2}(\$)$ & 253,742 & 257,694 & 258,106 & 259,479 & 261,023 \\
$z_{3}(\$)$ & 38,254 & 40,477 & 34,626 & 28,143 & 27,889 \\
Degree of satisfaction $(\%)$ & 87.54 & 86.68 & 85.84 & 85.02 & 84.26 \\
\hline
\end{tabular}

cost. Therefore, the DM selects the compensation coefficient $\gamma=0.1$ to maximize satisfaction. As displayed in Figures 5-7, as $\gamma$ increases, the degree of satisfaction with each objective decreases. The values of the objective functions $z_{1}$ and $z_{3}$ decrease as the compensation coefficient increases, but objective function $z_{2}$ increases. To optimize the outcome of the proposed FMOP model, the DM must select the weights of the objective functions according to his/her preferences and the most important parameters (upper 
Table 7. Comparison of solutions.

\begin{tabular}{ccc}
\hline Membership function & $\begin{array}{c}\text { Piecewise linear } \\
\text { membership function } \\
\text { (Hannan [32], 1981) }\end{array}$ & $\begin{array}{c}\text { S-curve membership } \\
\text { function (Vasant [9], 2003), } \\
\text { the proposed FMOP model }\end{array}$ \\
\hline$z_{1}(\$)$ & $4,201,272$ & $4,191,614$ \\
$z_{2}(\$)$ & 241,161 & 253,742 \\
$z_{3}(\$)$ & 71,673 & 38,254 \\
Satisfactory degree (\%) & $88.21 \%$ & $87.54 \%$ \\
\hline
\end{tabular}

Table 8. Objective value and degree of satisfaction obtained with various upper and lower bounds.

\begin{tabular}{ccc}
\hline Item & $\begin{array}{c}\text { Upper and lower } \\
\text { bounds by DM }\end{array}$ & $\begin{array}{c}\text { Upper and lower } \\
\text { bounds by the FMOP model }\end{array}$ \\
\hline$z_{1}(\$)$ & $(4,160,000,4,370,000)$ & $(4,153,672,4,398,375)$ \\
$z_{2}(\$)$ & $(220,000,340,000)$ & $(214,202,355,759)$ \\
$z_{3}(\$)$ & $(0,360000)$ & $(0,382,745)$ \\
Satisfactory degree $(\%)$ & $88.21 \%$ & $89.02 \%$ \\
\hline
\end{tabular}

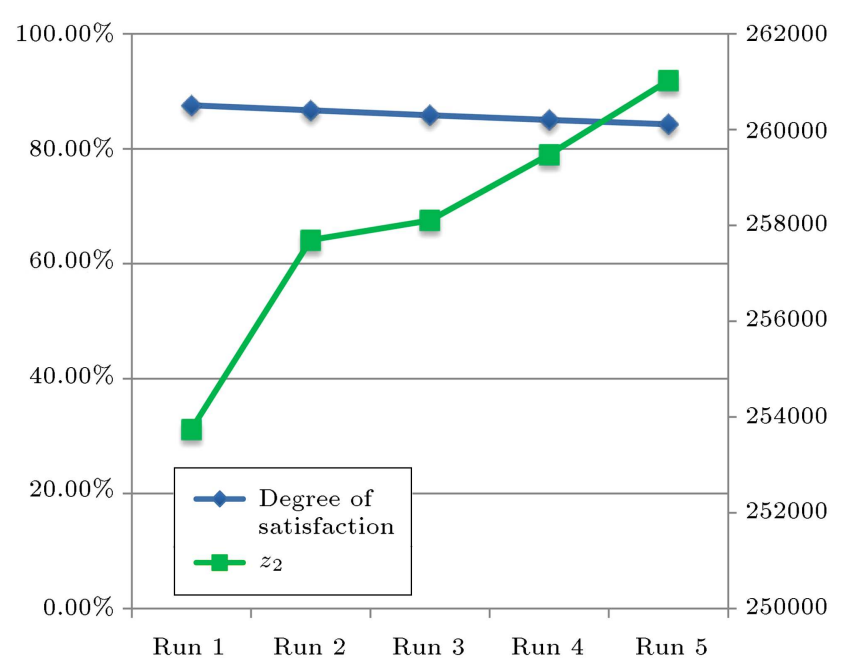

Figure 6. Inventory costs and degree of satisfaction for various $\gamma$ values.

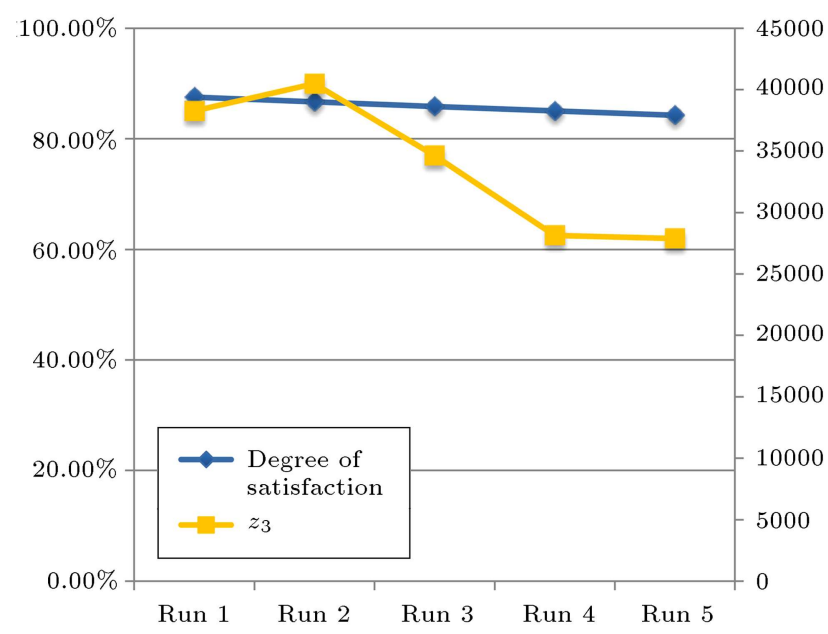

Figure 7. Penalty costs and degree of satisfaction for various $\gamma$ values. and lower bounds, compensation coefficient $(\gamma), \ldots)$ when S-curve membership functions are utilized.

\subsection{Computational analysis}

Table 7 compares the results obtained using the piecewise linear membership function with those obtained using the proposed model. The compromise solution obtained by using the piecewise linear membership function to minimize the total manufacturing cost, inventory holding cost, and penalty cost, simultaneously, is $z_{1}=\mathrm{NT} \$ 4,201,272, z_{2}=\mathrm{NT} \$ 241,161$, and $z_{3}=$ NT $\$ 71,673$, respectively, and the degree of satisfaction is $88.21 \%$. In contrast, the proposed model yields $z_{1}=$ $\mathrm{NT} \$ 4,191,614, z_{2}=\mathrm{NT} \$ 253,742$, and $z_{3}=\mathrm{NT} \$ 38,254$ and the degree of satisfaction is $87.54 \%$. In particular, the proposed FMOP model clearly yields lower total manufacturing cost and penalty cost than the piecewise linear membership function. Consequently, mobile phone production planning can be achieved using the proposed FMOP model with an acceptable degree of DM satisfaction.

Table 8 compares the results obtained by imposing upper and lower bounds using the proposed FMOP model. When the DM sets the upper and lower bounds, the degree of satisfaction is $88.21 \%$. In contrast, the proposed model yields a degree of satisfaction of $89.02 \%$. In particular, the proposed FMOP model yields a higher degree of satisfaction than what is achieved when the DM sets the upper and lower bounds. Therefore, the FMOP model is sufficiently flexible to provide various solutions that the DM can use to select the final preferred compromise solution.

This work concerns a real-work test case in Taiwan. Currently, company $\mathrm{Y}$ performs manual production planning, roughly estimating lot sizes for 
Table 9. Total manufacturing costs, inventory holding costs, and penalty costs for companies $\mathrm{Y}$ and $\mathrm{H}$.

\begin{tabular}{ccccc}
\hline \multicolumn{2}{c}{ Objective } & $\begin{array}{c}\text { Company Y (using } \\
\text { manual planning) }\end{array}$ & $\begin{array}{c}\text { Company H (using the } \\
\text { proposed FMOP model) }\end{array}$ & Improvement \\
\hline$z_{1}(\$)$ & $z_{1}^{\text {man }}$ & $4,485,966$ & - & $-332,294$ \\
& $z_{1}^{\text {opt }}$ & - & $4,153,672$ & \\
$z_{2}(\$)$ & $z_{2}^{\text {man }}$ & 335,194 & - & $-93,992$ \\
& $z_{2}^{\text {opt }}$ & - & 214,202 & \\
& & & - & $-187,748$ \\
$z_{3}(\$)$ & $z_{3}^{\text {man }}$ & 187,748 & 0 & \\
\hline
\end{tabular}

both manufactures and outsourced components in period. Table 9 compares the total manufacturing costs, inventory holding costs, and penalty costs obtained by companies $\mathrm{Y}$ (using manual planning) and $\mathrm{H}$ (using the proposed FMOP model), where $z_{1}^{\operatorname{man}}$ is the total manufacturing cost for company $\mathrm{Y}$ when lot sizes are set manually, and $z_{1}^{\text {opt }}$ is the total manufacturing cost for company $\mathrm{H}$ when lot sizes are optimized; $z_{2}^{\operatorname{man}}$ is the inventory holding cost for company $\mathrm{Y}$ when lot sizes are set manually, and $z_{2}^{\text {opt }}$ is the inventory holding cost for company $\mathrm{H}$ when lot sizes are optimized; $z_{3}^{\operatorname{man}}$ is the penalty cost for company $\mathrm{Y}$ when lot sizes are set manually, and $z_{3}^{\text {opt }}$ is the penalty cost for company $\mathrm{H}$ when lot sizes are optimized.

The total manufacturing costs when lot sizes are set manually and optimized are $\mathrm{NT} \$ 4,485,966$ and NT $\$ 4,153,672$, respectively. The inventory holding costs when lot sizes are set manually and optimized are $\mathrm{NT} \$ 335,194$ and $\mathrm{NT} \$ 214,202$, respectively. The penalty costs when lot sizes are set manually and optimized are $\mathrm{NT} \$ 187,748$ and $\mathrm{NT} \$ 0$, respectively. Therefore, the proposed FMOP model provides a practical means for solving production-planning decision problems of mobile phone manufacturing in fuzzy environments and it satisfies the practical requirements of planners.

\section{Conclusions}

This work presents a novel FMOP model with modified S-curve membership function to solve the production planning problem of mobile phone manufacturing with multiple components, multiple suppliers, and multiple periods in uncertain environments. The proposed FMOP model simultaneously minimizes overall manufacturing, total holding, and total penalty costs with manufacturer/supplier capacity and warehouse space constraints. Owing to its unique fuzzy mathematical programming method, the proposed model significantly contributes to efforts to solve production planning problems for mobile phone manufacturing in a fuzzy environment. Moreover, the proposed model provides a systematic decision-making procedure that allows a
DM to interactively adjust search directions until a satisfactory solution is obtained.

This work demonstrates the effectiveness of implementing the proposed model via an illustrative example of mobile phone manufacturing in Taiwan. Analytical results demonstrate that the lot-sizing decision can help DMs in production planning under fuzzy environments and satisfy managerial requirements. Importantly, the proposed model is a flexible means of setting parameters in decision models used for mobile phone manufacturing. Furthermore, the proposed model is highly promising for use in a component with similar mobile phone production planning requirements.

We recommend that future research extend the proposed model to evaluate production planning decisions involving different cases of demand uncertainty, supply quantity, and other cost components. Future research can also incorporate the proposed FMOP model and fuzzy set theory into a lot-sizing problem with different probability distributions for component yield in fuzzy environments. If transportation costs for mobile phone manufacturing are significant, then those used in this work may not be valid. Therefore, this work could be extended by developing an FMOP model that includes significant transportation costs.

\section{Acknowledgement}

The authors would like to thank the National Science Council of the Republic of China, Taiwan, for financially/partially supporting this research under Contract No. NSC 101-2221-E-020-017.

\section{References}

1. Tyagi, S., Yang, K., Tyagi, A. and Verma, A. "A fuzzy goal programming approach for optimal product family design of mobile phones and multiple-platform architecture", IEEE Transactions on Systems, Man, and Cybernetics-Part C: Applications and Reviews, 42, pp. 1519-1530 (2012).

2. Gules, H.K. and Burgess, T.F. "Manufacturing tech- 
nology and the supply chain", European Journal of Purchasing and Supply Management, 2, pp. 31-38 (1996).

3. Spekman, R.E., Kamauff, J. and Spear, J. "Towards more effective sourcing and supplier management", European Journal of Purchasing and Supply Management, 5, pp. 103-116 (1999).

4. Wang, E.J., Chen, Y.C., Wang, W.S. and Su, T.S. "Analysis of outsourcing cost-effectiveness using linear programming model with fuzzy multiple goals", International Journal of Production Research, 48, pp. 501$523(2010)$.

5. Chang, P.C., Lin, J.J. and Dzan, W.Y. "Forecasting of manufacturing cost in mobile phone products by casebased reasoning and artificial neural network models", Journal of Intelligent Manufacturing, 23, pp. 517-531 (2012).

6. Franke, C., Basdere, B., Ciupek, M. and Seliger, S. "Remanufacturing of mobile phones-capacity, program and facility adaptation planning", Omega: The International Journal of Management Science, 34, pp. 562570 (2006).

7. Peidro, D., Mula, J., Alemany, M.M.E. and Larío, F.C. "Fuzzy multi-objective optimisation for master planning in a ceramic supply chain", International Journal of Production Research, 50, pp. 3011-3020 (2012).

8. Díaz-Mdroñero, M., Peidro, D. and Vasant, P. "Vendor selection problem by using an interactive fuzzy multiobjective approach with modified S-cure membership functions", Computers and Mathematics with Applications, 60, pp. 1038-1048 (2010).

9. Vasant, P.M. "Application of fuzzy linear programming in production planning", Fuzzy Optimization and Decision Making, 2, pp. 229-241 (2003).

10. Vasant, P.M. "Solving fuzzy linear programming problems with modified S-curve membership function", International Journal of Uncertainty, Fuzziness and Knowledge-Based System, 13, pp. 97-109 (2005).

11. Vasant, P.M., Nagarajan, R. and Yaacob, S. "Decision making using modified s-curve membership function in fuzzy linear programming problem", Journal of Information and Communication Technology, 1, pp. 116 (2003).

12. Lee, S.J. and Lee, B.Y. "Case study of Samsung's mobile phone business", KDI School of Pub Policy \& Management Paper, pp. 4-11 (2004).

13. Heikkilä, J. and Cordon, C. "Outsourcing: a core or non-core strategic management decision", Strategic Change, 11, pp. 183-193 (2002).

14. Wang, E.J., Su, T.S. and Liu, J.Y. "Fuzzy multiple goal programming for analyzing outsourcing costeffectiveness in hi-tech manufacturing", International Journal of Production Research, 51, pp. 3920-3944 (2013).
15. Vollmann, T., Cordon, C. and Heikkilä, J. "Teaching supply chain management to business executives", Production and Operations Management, 9, pp. 81-90 (2000).

16. Shy, O. and Stenbacka, R. "Strategic outsourcing", Journal of Economic Behavior \& Organization, 50, pp. 203-224 (2003).

17. Alvarez, L.H.R. and Stenbacka, R. "Partial outsourcing: A real options perspective", International Journal of Industrial Organization, 25, pp. 91-102 (2007).

18. Wilde-Ramsing, J. and Haan, E., The High Cost of Calling: Critical Issues in the Mobile Phone Industry, Available at SSRN: 1660422, pp. 39-62 (2006).

19. Olhager, J., Persson, F., Berne, P. and Rosén, S. "Supply chain impacts at Ericsson-from production units to demand-driven supply units", International Journal of Technology Management, 23, pp. 40-59 (2002).

20. Xiong, G., Fan, D., Liu, S., Nyberg, T.R. and Wang, F.Y. "Mass customization manufacturing solution for cell phone production", IEEE International Conference on Automation and Logistics (ICAL), August 1620, Hong Kong and Macau, pp. 518-523 (2010).

21. Zadeh, L.A. "Fuzzy set", Information and Control, 8, pp. 38-53 (1965).

22. Bellman, R.E. and Zadeh, L.A. "Decision-making in a fuzzy environment", Management Science, 17, pp. 141-164 (1970).

23. Zimmermann, H.J. "Fuzzy programming and linear programming with several objective functions", Fuzzy Sets and Systems, 1, pp. 45-55 (1978).

24. Peidro, D. and Vasant, P. "Transportation planning with modified S-curve membership functions using an interactive fuzzy multi- objective approach", Applied Soft Computing, 11, pp. 2656-2663 (2011).

25. Ganesan, T., Vasant, P. and Elamvazuthi, I. "Hybrid neuro-swarm optimization approach for design of distributed generation power systems", Neural Comput \& Applic, 23, pp. 105-117 (2013).

26. Jiménez, F., Sánchez, G. and Vasant, P. "A multiobjective evolutionary approach for fuzzy optimization in production planning", Journal of Intelligent \& Fuzzy Systems, 25, pp. 441-455 (2013).

27. Vasant, P. "Hybrid LS-SA-PS methods for solving fuzzy non-linear programming problems", Mathematical and Computer Modelling, 57, pp. 180-188 (2013).

28. Nowakowska, M. "Methodological problems of measurement of fuzzy concepts in the social sciences", Behavioral Science, 22, pp. 107-115 (1977).

29. Torabi, S.A. and Hassini, E. "An interactive possibilistic programming approach for multiple objective supply chain master planning", Fuzzy Sets and Systems, 159, pp. 193-214 (2008).

30. Lai, Y. and Hwang, C. "Possibilistic linear programming for managing interest rate risk", Fuzzy Sets and Systems, 54, pp. 135-146 (1993). 
31. Selim, H. and Ozkarahan, I. "A supply chain distribution network design model: an interactive fuzzy goal programming-based solution approach", The International Journal of Advanced Manufacturing Technology, 36, pp. 401-418 (2008).

32. Hannan, E.L. "Linear programming with multiple fuzzy goals", Fuzzy Sets and Systems, 6, pp. 235-248 (1981).

\section{Biographies}

Tai-Sheng Su is an Associate Professor in the Department of Industrial Management at National Pingtung University of Science and Technology in Taiwan, ROC. $\mathrm{He}$ received a $\mathrm{PhD}$ degree in the Department of Industrial Engineering and Management from National Chiao Tung University in Taiwan. His research areas include production management, operations manage- ment, and logistics management. He has published articles in Computers \& Industrial Engineering, International Journal of Production Research, and Journal of Manufacturing Systems.

Chin-Chun $\mathbf{W u}$ is an Assistant Professor in the Department of Information Management at I-Shou University, Taiwan. She received her $\mathrm{PhD}$ degree in the College of Management from National Yunlin University of Science and Technology. Her research activities include applications of decision analysis, quality engineering, statistics, and mathematical economics.

Chih-Hsiu Lin is a Master's student in the Department of Industrial Management at National Pingtung University of Science and Technology in Taiwan. His research areas include supply chain management and production management. 